\title{
OPTIMAL GLOBAL CONFORMAL SURFACE PARAMETERIZATION FOR VISUALIZATION*
}

\author{
MIAO JIN ${ }^{\dagger}$, YALIN WANG ${ }^{\ddagger}$, XIANFENG GU ${ }^{\S}$, AND SHING-TUNG YAU ${ }^{\llbracket l}$
}

\begin{abstract}
All orientable metric surfaces are Riemann surfaces and admit global conformal parameterizations. Riemann surface structure is a fundamental structure and governs many natural physical phenomena, such as heat diffusion, electric-magnetic fields on the surface. Good parameterization is crucial for simulation and visualization. This paper gives an explicit method for finding optimal global conformal parameterizations of arbitrary surfaces. It relies on certain holomorphic differential forms and conformal mappings from differential geometry and Riemann surface theories. Algorithms are developed to modify topology, locate zero points, and determine cohomology types of differential forms. The implementation is based on finite dimensional optimization method. The optimal parameterization is intrinsic to the geometry, preserving angular structure, and can play an important role in various applications including texture mapping, remeshing, morphing and simulation. The method is demonstrated by visualizing the Riemann surface structure of real surfaces represented as triangle meshes.
\end{abstract}

Key words: Computational geometry and object modeling; Curve, surface, solid, and object representations; Surface parameterization.

1. Introduction. Surface parameterization is the process of mapping a surface to a planar domain. Surface parameterization has many applications in various fields of science and engineering, including texture mapping, geometric morphing, surface matching, surface remeshing, and surface extrapolation. For example, texture mapping can be used to enhance the visual quality and generate different visual results. Geometric morphing can be used to generate vivid animation results. Essentially, surface parametrization can convert 3D geometric problem to $2 \mathrm{D}$ ones, thereby improving the efficiency and simplifying the computation.

Conformal surface parameterizations have many merits, such as preserving angular structure, being intrinsic to geometry, stable with respect to different triangulations and small deformations. It has been widely used for many applications, such as non-distorted texture mapping [ Lévy et al. 2002], [ Haker et al. 2000], [ Kraevoy et al. 2003], surface remeshing [ Alliez et al. 2002], surface fairing [ Lévy 2003], surface matching [ Gu and Yau 2002], brain mapping [ Angenent et al. 1999], [ Gu et al. 2003] etc.

It is desirable to parameterize surfaces globally without any seams. The existence of global conformal parameterization is a non-trivial fact. This is equivalent to that all orientable surfaces are Riemann surfaces. The atlas formed by the global conformal parameterization is the so-called conformal structure. Conformal structure is a fundamental structure between metric structure and topological structure and governs many natural physical phenomena.

\footnotetext{
${ }^{*}$ Received on May 15, 2004; accepted for publication on November 15, 2004.

${ }^{\dagger}$ Computer Science Department, State University of New York at Stony Brook, Stony Brook, New York 117944400. E-mail: mjin@cs.sunysb.edu

¥Department of Mathematics, UCLA, Los Angeles, CA 90095. E-mail: ylwang@math.ucla.edu

${ }^{\S}$ Computer Science Department, State University of New York at Stony Brook, Stony Brook, New York 117944400. E-mail: gu@cs.sunysb.edu

IDepartment of Mathematics, Harvard University, Cambridge, MA 02138. E-mail: yau @ math.harvard.edu
} 

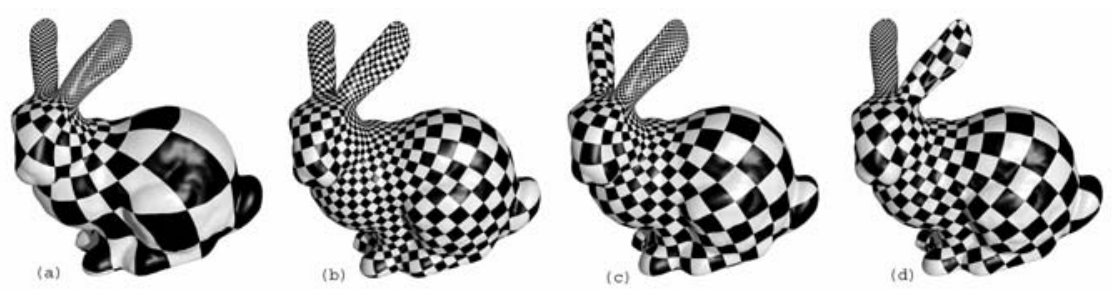

FIG. 1. Uniform global conformal parameterization $((a)$ and $(b))$ and region emphasized conformal parameterization $((c)$ and $(d))$. (a). Least uniform conformal parameterization with energy: 21.208e - 5. (b). Most uniform conformal parameterization with energy: $3.685 e-5 .(c)$. Maximizing the parameter area of the left half surface (with percentage: $83.48 \%$ ). (d). Maximizing the parameter area of the right half surface (with percentage: $82.58 \%$.)

The abstract concept of Riemann surface can also be visualized by texture mapping special pattern using global conformal parameterizations. This is the only means to visually convey conformal information of surfaces.

The early work of global conformal parameterization has been done in [Gu and Yau 2002, $\mathrm{Gu}$ and Yao 2003], where the basis for all possible global conformal parameterizations are computed. Because global conformal parameterization is non-unique, the problem of finding the optimal one remains open.

This paper introduces an explicit method to find the optimal global conformal parameterizations of arbitrary surfaces. First, the metrics for measuring the quality of conformal parameterizations are designed. Second, the major factors affecting the quality of the parameterization are summarized. Then algorithms are developed to modify the topology, locate the zero points, and determine the cohomology types of the differential forms. The method is based on finite dimensional optimization and demonstrated by visualizing the Riemann surface structure of real surfaces.

1.1. Contributions. This paper introduces algorithms to optimize global conformal parameterizations. The method is based on Riemann surface theories and differential geometry, therefore it is rigorous and general. The optimization algorithms can be generalized to all parameterization methods based on convex combinations [ Floater and Hormann 2003].

We also address several open issues closely related to the parameterization optimization. They are listed as follows.

1. In [ Floater and Hormann 2003], the author raised the following open question: "Under what boundary condition a harmonic map between two topological disks is conformal?" We answer this question in an algorithmic way. We compute the double covering of a topological disk (double covering means glue two copies of the same surface along their boundaries to form a closed symmetric surface, details are described in [ Gu and Yau 2003]), and conformally map the double covering to a sphere preserving the symmetry. Thus the disk itself is mapped to a hemisphere. Then a conformal map between two disks is induced by their mappings to the same hemisphere. The boundary condition which makes a harmonic map to be conformal can be computed using this algorithm directly. 
2. The difference between the zero points of a conformal parameterization and singularities of general vector fields is that the zero points of a global conformal parameterization can not be arbitrarily assigned and they are determined by the conformal structure. To the best of our knowledge, this statement has never been addressed in computer graphics field although it is the major topological obstruction for any surface parameterization methods.

3. This paper proves the following fact: the area stretching factor increases exponentially at the tip of long tubes and it is true for all other parameterization methods. This shows the limits of current parameterization techniques and justifies topological modification techniques proposed in this paper. Although some researchers reached the same conclusion by heuristic method, a rigorous proof is given in this paper.

1.2. Related Work. Surface conformal parameterization algorithms have been thoroughly studied in the literature. We summarize them according to the topologies of surfaces that they can handle.

Conformal map for topological disks. Many researchers propose methods to build a conformal map for topological disks. Pinkall and Polthier derive the discrete Dirichlet energy in [ Pinkall and Polthier 1993]. Eck et al. [ Eck et al. 1995] introduce the discrete harmonic map, which approximates the continuous harmonic maps by minimizing a metric dispersion criterion. Duchamp formulates hierarchical harmonic embedding in [ Duchamp et al. 1997]. Floater introduces a shape-preserving method in [ Floater 1997], which is very similar to harmonic maps for planar surfaces. Sheffer and de Sturler introduce angle based flattening to compute conformal maps. Desbrun et al. [ Alliez et al. 2002, Desbrun et al. 2002] compute the discrete Dirichlet energy and apply conformal parameterization to interactive geometry remeshing. Levy et al.[ Lévy et al. 2002] compute a quasi-conformal parameterization by approximating the Cauchy-Riemann equation using the least square method. Hormann and Greiner propose the MIPS parameterization [ Hormann and Greiner 1999], which roughly attempts to preserve the ratio of singular values over the parameterization. Degener et al. [ Degener et al. 2003] extends the method [ Hormann and Greiner 1999] and provides a control parameter that allows to mediate between angle and area distortion.

Conformal map for genus zero closed surfaces. Haker et al. [ Haker et al. 2000] introduce a method to compute a global conformal mapping from a genus zero surface to a sphere by representing the Laplace-Beltrami operator as a linear system. Gu et al. [Gu and Yau 2002] introduce a non-linear optimization method to compute global conformal parameterizations for genus zero surfaces. The optimization is carried out in the tangential spaces of a sphere.

Conformal map for high genus surfaces. Few researchers report their work on surfaces with complicated topology. Gu and Yau introduce algorithms to compute conformal structures determined by the metric for general closed surfaces in [ Gu and Yau 2002]. The proposed method approximates De Rham cohomology by simplicial cohomology, and computes a basis of holomorphic 1-forms. Later the method is generalized for surfaces with boundaries 
in [ Gu and Yau 2003].

In [ Stephenson 1999, Mercat 2001], the Riemann surface structure are defined for combinatorial meshes. Because the metric information are ignored in their works, their methods cannot be applied to our problems.

2. Sketch of Mathematical Theories and Algorithm Overview. This section introduces the basic concepts in Riemann surface theory related to global conformal parameterization and an overview of the optimization algorithms.

2.1. Theoretic Background. The basic concepts of Riemann surface theories are briefly sketched. Further details can be found in [ Jost 1991], [ Griffiths and Harris 1978] and [Schoen and Yau 1997].

Conformal Chart. Let $U$ be an open set of $S \in \mathbb{R}^{3}$. A parameterization of $U$ is a one to one map $z: U \rightarrow \mathbb{R}^{2}$, which maps $U$ to the $(u, v)$ plane. $(U, z)$ is called a chart of $S$. In the case of our conformal chart, the first fundamental form satisfies: $d s^{2}=\lambda(u, v)\left(d u^{2}+d v^{2}\right)$, where $\lambda(u, v)$ is called the stretch factor, a function that scales the metric at each point $(u, v)$. The coordinate pair $(u, v)$ is called a conformal parameter of the surface patch $U .(U, z)$ is called a conformal chart of $S$.

Conformal Atlas. All oriented metric surfaces are Riemann surfaces and have a global conformal atlas, or a set of conformal charts. In the following discussion, we treat $\mathbb{R}^{2}$ as a complex plane, where the point $(u, v)$ is equivalent to $z=u+i v$, and $(u,-v)$ is equivalent to $\bar{z}=u-i v$. In later sections, we use both representations interchangeably.

Let $S$ be a surface in $\mathbb{R}^{3}$ with an atlas $\left\{\left(U_{\alpha}, z_{\alpha}\right)\right\}$, where $\left(U_{\alpha}, z_{\alpha}\right)$ is a chart, and $z_{\alpha}$ : $U_{\alpha} \rightarrow \mathbb{C}$ maps an open set $U_{\alpha} \subset S$ to the complex plane $\mathbb{C}$.

The atlas is called conformal if (1). each chart $\left(U_{\alpha}, z_{\alpha}\right)$ is a conformal chart. Namely, on each chart, the first fundamental form can be formulated as $d s^{2}=\lambda\left(z_{\alpha}\right) d z_{\alpha} d z_{\alpha}^{-}$, (2). the transition maps $z_{\beta} \circ z_{\alpha}^{-1}: z_{\alpha}\left(U_{\alpha} \cap U_{\beta}\right) \rightarrow z_{\beta}\left(U_{\alpha} \cap U_{\beta}\right)$ are holomorphic.

A chart is compatible with a given conformal atlas if adding it to the atlas again yields a conformal atlas. A conformal structure ( Riemann surface structure ) is obtained by adding all compatible charts to a conformal atlas. A Riemann surface is a surface with a conformal structure.

Holomorphic 1-form. Given a Riemann surface $S$ with a conformal atlas $\left\{\left(U_{\alpha}, z_{\alpha}\right)\right\}$, a differential 1-form $\omega$ is defined by a family $\left\{\left(U_{\alpha}, z_{\alpha}, \omega_{\alpha}\right)\right\}$, such that (1). $\omega_{\alpha}=f_{\alpha}\left(z_{\alpha}\right) d z_{\alpha}$, where $f_{\alpha}$ is holomorphic on $U_{\alpha}$. (2). if $z_{\alpha}=\phi_{\alpha \beta}\left(z_{\beta}\right)$ is the coordinate transformation on $U_{\alpha} \cap U_{\beta}(\neq \emptyset)$, then $f_{\alpha}\left(z_{\alpha}\right) \frac{d z_{\alpha}}{d z_{\beta}}=f_{\beta}\left(z_{\beta}\right)$, the local representation of the differential form $\omega$ satisfies the chain rule.

For a Riemann surface $S$ with genus $g>0$, all holomorphic 1-forms on $S$ form a complex $g$-dimensional vector space (real $2 g$ dimension), denoted as $\Omega^{1}(S)$. The quality of a global conformal parameterization for a high genus surface is mainly determined by the choice of the holomorphic 1-form. 
The zero points of a holomorphic 1-form $\omega$ are the points where, on any local representation, $\left(U_{\alpha}, z_{\alpha}, \omega_{\alpha}\right)$ equals to zero. For a genus $g>0$ surface, there are in general $2 g-2$ zero points for each holomorphic 1-form.

Möbius Transformation Group. For genus zero closed surfaces, there is no holomorphic one form. The global conformal parameterization is a conformal map $\phi: S \rightarrow S^{2}$ from the surface $S$ to the unit sphere $S^{2}$. Two such kinds of transformations differ by a Möbius transformation on $S^{2}$. Suppose both $\phi_{1}$ and $\phi_{2}$ are two conformal parameterizations of $S$, consequently $\phi_{2} \circ \phi_{1}^{-1}=\mu$, where $\mu$ is a Möbius automorphism of the sphere. All conformal maps from $S$ to $S^{2}$ can then be formulated as $\mu \circ \phi_{1}$. We compute one conformal map $\phi_{1}$ first, then compose it with a Möbius transformation $\mu$. By choosing appropriate $\mu$, we can optimize the energy.

A genus zero open surface can be globally conformally parameterized by the unit disk. Two such kinds of parameterizations differ by a Möbius transformation defined on the disk. We can find the best one with a similar method to that is used for a genus zero closed surface.

2.2. Optimization Algorithms Overview. In order to measure the quality of a global conformal parameterization, we define different metrics for different applications. There are several main factors affecting the quality of a parameterization, including the topology of the surface, the zero point position and the choice of the holomorphic 1-form for a high genus surface or the Möbius transformation for a genus zero surface. The algorithms for optimizing these factors are as the following

- Metric for parameterization. We formulate different functionals to measure the qualities of parameterizations, including uniformity energy, parameter area of emphasized regions and zero points locations.

- Topology Optimization. The long tube shape causes exponential shrinking parameterization. We design method to mediate this problem.

- Zero Point Allocation. The parameterizations near the zero points are singular, it is desirable to allocate zero points at the predefined positions.

- Optimal Holomorphic 1-form. The global conformal parameterization for a higher genus surface is induced by a holomorphic 1 -form. The special holomorphic 1-form is chosen to optimize the functional for parameterizations.

- Optimal Möbius Transform. The global conformal parameterization of a genus zero surface is determined by a Möbius transform. The algorithm is designed to find an optimal Möbius transform to maximize the functional.

2.3. Approximation Strategy. The concepts of Riemann surfaces are defined for smooth surfaces. In practice, it is impossible to represent the smooth surface and conformal structure using finite memory. We approximate them by the finite element method. Specifically, we approximate a smooth surface $S$ using a series of piecewise linear triangular meshes $\left\{\tilde{S}_{n}\right\}$ and approximate the smooth conformal structures of $S$ using piecewise linear mappings defined on $\left\{\tilde{S}_{n}\right\}$ also. Such kind of discrete mappings are called as discrete holomorphic 1- 
forms.

A natural question arises whether the approximation converges to the real conformal structure of the smooth surface. The answer is positive. Computing conformal structure is equivalent to solve an elliptic Partial Differential Equation (PDE) on the surface. It has been proven in finite element field that the discrete approximation converges to the real solution [ Ciarlet 1978]. Also, the solutions to elliptic PDEs are stable and smooth in general. This implies the convergence and stability of our approximation.

Because of the convergence and the stability of our discrete approximations, they behave like the real solutions asymptotically. In the following discussion, we conceptually treat them as smooth solutions and do not differentiate discrete approximation and smooth solution.

There is an important point we want to clarify. The conformal structure is determined by the metric of the surface. Even if a surface is not smooth, such as the mesh $\tilde{S}_{n}$ in the approximation, it still has smooth metric and smooth conformal structure. The discrete holomorphic 1-forms in the approximations are not the real conformal structure of the mesh $\tilde{S}_{n}$.

3. Algorithms for Global Conformal Parameterization Optimization. In our current work, the surfaces are represented as meshes. Suppose $K$ is a simplicial complex, and a mapping $r:|K| \rightarrow R^{3}$ embeds $|K|$ in $R^{3} . M=(K, r)$ is called a triangular mesh. $K_{n}$ where $n=0,1,2$ are the sets of $n$-simplices. We use $\left[v_{0}, v_{1}, \cdots, v_{n}\right]$ to denote a $n$-simplex, where $v_{i} \in K_{0}$.

We use the following symbols in the following discussion: $E$ - energy for a parameterization, $\omega$ - a holomorphic 1-form, $\lambda_{i}$ - the coefficients of $\omega, \lambda$ - conformal factor, $\tau$ the stereo-graphic projection, $\mu$ - a Möbius transformation, $\phi$ - a conformal map between surfaces.

3.1. Computing Conformal Structures. We use the methods introduced in [Gu and Yau 2002], [ Gu and Yau 2003] to compute conformal structures.

Genus 0 closed surfaces, they can be conformally parameterized by a unit sphere. For genus 0 surfaces, Harmonic maps are equivalent to conformal maps. We use a Gauss map as the initial map, then we use the heat flow method to reduce the harmonic energy with special constraints. The final harmonic map is a global conformal parameterization. By composing with a Möbius map of the sphere, we can obtain all possible global conformal parameterizations.

For genus 0 open surfaces, we double cover it and get a closed symmetric surface. We can map this double covered surface conformally to a sphere and preserve the symmetry; i.e, each copy of the original surface is mapped to a hemisphere. Then we use stereo-graphic projection to map a hemisphere to a unit disk; the surface is globally conformally parameterized by the disk. By composing with a Möbius map of the disk, we can construct all global conformal parameterizations for the surface.

For higher genus surfaces, their conformal structures can be represented as a holomorphic one-form basis, which are $2 g$ functions $\omega_{i}: K_{1} \rightarrow R^{2}, i=1,2 \cdots, 2 g$. Any holomorphic one 
form $\omega$ is a linear combination of these functions. The surface can be cut open to a topological disk; namely a fundamental domain. By integrating $\omega$ on a fundamental domain, the whole surface can be globally conformally mapped to the $u v$ plane.

The computation process for $\left\{\omega_{i}, i=1,2, \cdots, 2 g\right\}$ can be summarized as computing homology basis, cohomology basis, harmonic one form basis and holomorphic one form basis.

For surfaces with boundaries, the double covering techniques are applied to convert them to closed symmetric surfaces. Therefore, in the following discussion, we assume the surfaces are closed.

3.2. Metrics for parameterization. In order to convert the whole mesh to a geometry image, or spline surface patches, parameterizations with high uniformity are preferred. It is often desirable to allocate more parameter areas for special regions on the surface in real applications. For example, in surface remeshing, more samples are required for regions with high Gaussian curvature or sharp features. Sometimes, multi-chart geometry images are used to represent the shape. We can use several global parameterizations. Each of them will emphasize a surface region and convert it to one chart in the geometry image. In this scenario, the parameterization emphasizing different regions are also desirable. For high genus surfaces, the existence of zero points are unavoidable, the neighborhoods of zero points will be under sampled in the parameter domain. Therefore users would like to assign the zero points at positions which are with lower curvature or less visible. In order to allocate zero points at the prescribed positions, we design a special metric to measure the parameter area of the neighborhoods of the given points. If the parameterization with zero points at the desired position, this metric will be close to zero.

Suppose $\Omega \subset R^{2}$ is the parameter domain for a surface $S$ and $(u, v)$ are parameters on $\Omega$, then the functional for measuring uniformity is

$$
E=\int_{\Omega}\left(\lambda(u, v)^{2}-1\right)^{2} d u d v
$$

where $\lambda$ is the conformal factor, subject to

$$
\int_{\Omega} d u d v=\int_{\Omega} \lambda(u, v)^{2} d u d v
$$

Similarly, suppose $\Omega$ is divided into two regions $\Omega_{1}$ and $\Omega_{2}$, we would like to emphasize $\omega_{1}$, then the functional is

$$
E=\int_{\Omega_{1}} \lambda(u, v)^{2} d u d v
$$

subject to

$$
\int_{\Omega_{1} \cup \Omega_{2}} \lambda(u, v)^{2} d u d v=\int_{\Omega} d u d v
$$


For high genus surfaces, if we want to assign zero points for a global conformal parameterization, different functionals should be formulated to minimize the conformal factor at the desired points. Suppose we want to assign $\left\{p_{1}, p_{2}, \cdots, p_{n}\right\} \subset S$ as zero points, $U_{i} \subset \Omega$ is a neighborhood of $p_{i}$, and $\omega$ is a holomorphic 1 -form, we define the functional as

$$
E(\omega)=\cup_{i=1}^{n} \int_{U_{i}} \omega \wedge \bar{\omega} .
$$

Intuitively, this functional measures the area of the neighborhoods of zero points on the parameter domain. If there is a holomorphic 1-form $\omega$ with zero points at $p_{i}$ 's, then its $E(\omega)$ should be zero.

3.3. Optimal Holomorphic 1-form for High Genus Surface. A global conformal parameterization for a high genus surface can be obtained by integrating a holomorphic one form $\omega$. Suppose $\left\{\omega_{i}, i=1,2, \cdots, 2 g\right\}$ is a holomorphic 1 -form basis, arbitrary holomorphic 1-form has the formulae $\omega=\Sigma_{i=1}^{2 g} \lambda_{i} \omega_{i}$. The energy for the parameterization is denoted as $E(\omega)$, which is a function of the linear combination coefficients $\lambda_{i}$. The necessary condition for the optimal holomorphic 1 -form is straight forward, $\frac{\partial E}{\partial \lambda_{i}}=0, i=1,2, \cdots, 2 g$. If the Hessian matrix $\left(\frac{\partial^{2} E}{\partial \lambda_{i} \partial \lambda_{j}}\right)$ is positive definite, then $E$ will reach the minimum. If the Hessian matrix is negative definite, $E$ will be maximized. Traditional Newton's method can be applied for the optimization with the constraint that the total area in the parameter domain is fixed.

3.3.1. Uniform Global Conformal Parameterization. Given any holomorphic oneform $\omega, \omega=\sum_{k=1}^{2 g} \lambda_{k} \omega_{k}$, we require the total parameter area equal to the total area of the surface in $R^{3} 2$,

$$
\sum_{\left[v_{0}, v_{1}, v_{2}\right] \in K_{2}} \frac{1}{2}\left|\omega\left(\left[v_{0}, v_{1}\right]\right) \times \omega\left(\left[v_{1}, v_{2}\right]\right)\right|=\sum_{\left[v_{0}, v_{1}, v_{2}\right] \in K_{2}} S_{\left[v_{0}, v_{1}, v_{2}\right]}
$$

where $S_{\left[v_{0}, v_{1}, v_{2}\right]}$ is the area of face $\left[v_{0}, v_{1}, v_{2}\right]$ in $R^{3}$. The uniformity functional 1 is defined as the sum of the squared area differences of faces,

$$
E(\omega)=\sum_{\left[v_{0}, v_{1}, v_{2}\right] \in K_{2}}\left(\frac{1}{2}\left|\omega\left(\left[v_{0}, v_{1}\right]\right) \times \omega\left(\left[v_{1}, v_{2}\right]\right)\right|-S_{\left[v_{0}, v_{1}, v_{2}\right]}\right)^{2} .
$$

Both the constraint and the energy functional are polynomials with respect to $\lambda_{i}$ 's. For example, the constraint can be reformulated as a quadratic form; let $c_{i, j}=\sum_{\left[v_{0}, v_{1}, v_{2}\right] \in K_{2}} \frac{1}{2} \mid \omega_{i}\left(\left[v_{0}\right.\right.$, $\left.\left.v_{1}\right]\right) \times \omega_{j}\left(\left[v_{1}, v_{2}\right]\right) \mid$, then the constraint is $\sum_{i, j=1}^{2 g} c_{i j} \lambda_{i} \lambda_{j}=$ const.

We use Newton's method to optimize the energy with constraints. Because the energy is quintic of degree 4 , the extremal points are not unique. We randomly choose initial values for $\lambda_{i}$ 's, and choose the global optimal solution from local optimal ones. By minimizing the energy, we get the most uniform parameterization, for the purpose of comparison, we get the least uniform parameterization by maximizing the energy.

Figure 1 and 2 demonstrate the computation results. The least uniform and the most 
uniform global parameterization are illustrated by using a checkerboard-texture map. Figure 5 using the grid pattern to illustrate the computation results.

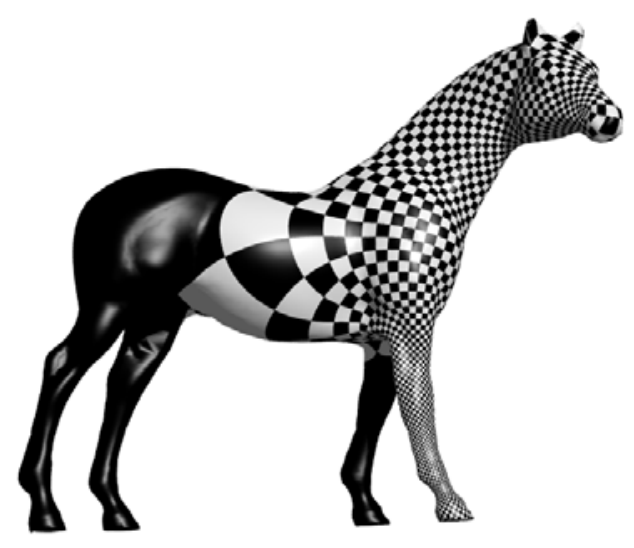

a. Least Uniform

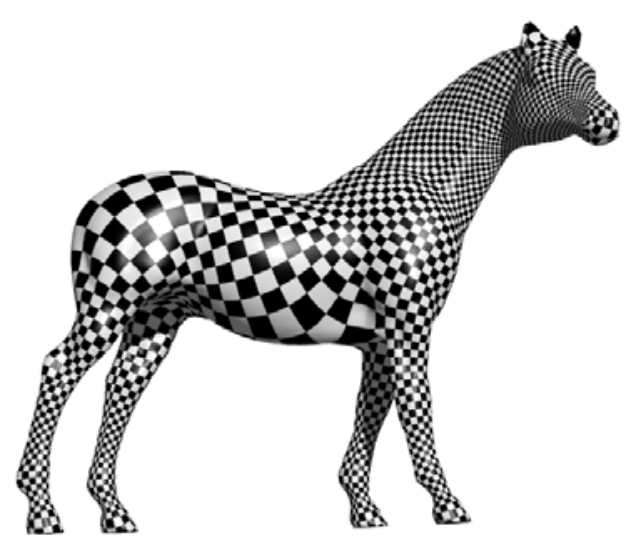

b. Most Uniform

FIG. 2. Uniform Global Conformal Parameterization. Least uniform conformal parameterization, energy: 16.983e-5 (a). Most uniform conformal parameterization, energy: $7.878 e-5(b)$.

3.3.2. Emphasized Global Conformal Parameterization. Suppose we subdivide the whole surface into two regions $D_{0}$ and $D_{1} . D_{0}$ and $D_{1}$ may be disconnected, with complicated topologies, and we want to maximize the parameter areas for $D_{0}$. Then we define the emphasized area energy 3 as,

$$
E(\omega)=\frac{1}{2} \sum_{\left[v_{0}, v_{1}, v_{2}\right] \in D_{0}}\left|\omega\left(\left[v_{0}, v_{1}\right]\right) \times \omega\left(\left[v_{1}, v_{2}\right]\right)\right|
$$

with the same constraint in equation 6 .

The functional can be represented as a quadratic form directly. Let $c_{i, j}=\sum_{\left[v_{0}, v_{1}, v_{2}\right] \in D_{0}}$ $\left|\omega_{i}\left(\left[v_{0}, v_{2}\right]\right) \times \omega_{j}\left(\left[v_{1}, v_{2}\right]\right)\right|$, then the emphasized area energy is

$$
E\left(\lambda_{1}, \lambda_{2}, \cdots, \lambda_{2 g}\right)=\sum_{i, j=1}^{2 g} c_{i j} \lambda_{i} \lambda_{j} .
$$

By maximizing this functional, we get more samples on $D_{0}$ and less samples on $D_{1}$, and vice versa. The critical point is unique in general cases. We use Newton's method for the optimization with arbitrary initial values for $\lambda_{i}$ 's.

Figure 1 demonstrates the optimization of the emphasized area energy for the bunny surface model. The surface is equally subdivided into the left part and the right part. Figure 1 (a) emphasizes the left part, and the parameter area of the left part is $83.48 \%$ of the total parameter area. Figure 1 (b) emphasizes the right part, the parameter area is $82.58 \%$ of the total parameter area. 
3.4. Optimal Möbius Transform for Genus Zero Surface. For genus zero surfaces, there are no holomorphic one forms. We conformally map the surface to a unit sphere or a unit disk. Because the parameter domains are fixed, the constraint 6 is unnecessary.

We can still use the uniformity energy or the emphasized area energy, but the admissible transformations are changed to the Möbius transformations.

Topological sphere. The Möbius transformation on the complex plane has the formula $\mu(z)=\frac{a z+b}{c z+d}, a d-b c=1, a, b, c, d \in \mathbb{C}$. A sphere can be conformally mapped to the complex plane by a stereographic projection $\tau: S^{2} \rightarrow \mathbb{C}, \tau(x, y, z)=\frac{x}{1-z}+\sqrt{-1} \frac{y}{1-z}$.

A conformal automorphism $\phi$ of the sphere can be formulated as $\phi=\tau^{-1} \circ \mu \circ \tau$,

We first compute a conformal map $\phi_{0}: S \rightarrow S^{2}$ from the surface to the sphere, all admissible conformal mappings can be represented as $\phi_{\mu}=\tau^{-1} \circ \mu \circ \tau \circ \phi_{0}$.

The uniformity functional becomes

$$
E(\mu)=\sum_{\left[v_{0}, v_{1}, v_{2}\right] \in K_{2}}\left(\left|\phi_{\mu}\left(v_{0}\right), \phi_{\mu}\left(v_{1}\right), \phi_{\mu}\left(v_{2}\right)\right|-\left|r\left(v_{0}\right), r\left(v_{1}\right), r\left(v_{2}\right)\right|\right)^{2}
$$

where $|a, b, c|$ represents the area of the triangle formed by $a, b, c$. This is a rational formula with respect to the coefficients of $\mu$. We use Newton's method to optimize it without any constraints.

Similarly, the emphasized area energy is formulated by

$$
E(\mu)=\sum_{\left[v_{0}, v_{1}, v_{2}\right] \in D_{0}}\left|\phi_{\mu}\left(v_{0}\right), \phi_{\mu}\left(v_{1}\right), \phi_{\mu}\left(v_{2}\right)\right| .
$$

We use Newton's method to maximize the energy. Because the optimal solutions are not unique, we randomly choose the initial Möbius transformation $\mu_{0}$, and use $\phi_{\mu_{0}}$ as the initial parameterization.

Topological disk. For the topological disk case, we use double covering to make it a symmetric topological sphere. But we restrict the admissible transformations to be in a subgroup of the Möbius group, which preserves the symmetry; namely $\mu(\bar{z})=\overline{\mu(z)}$.

The formula for such a Möbius transformation can be written as $\mu(z)=\frac{a z+b}{b z+\bar{a}}, a \bar{a}-b \bar{b}=$ $1, a, b \in \mathbb{C}$.

Other steps are similar to those for the case of a topological sphere. Figure 3 illustrates a Möbius transformation from the disk to itself.

3.5. Topological Optimization. In this section, we introduce an automatic method to modify the topology of the surface to improve the uniformity of the parameterization.

For long tube shapes, such as fingers and tails, the area distortion is usually very big. We want to show that the problem cannot be solved by linear combination of the holomorphic one-form basis. We have to modify the conformal structure of the surface itself; namely, we either change the Riemannian metric, or modify the topology.

First we will show the fact that the conformal factor will increase exponentially on long 

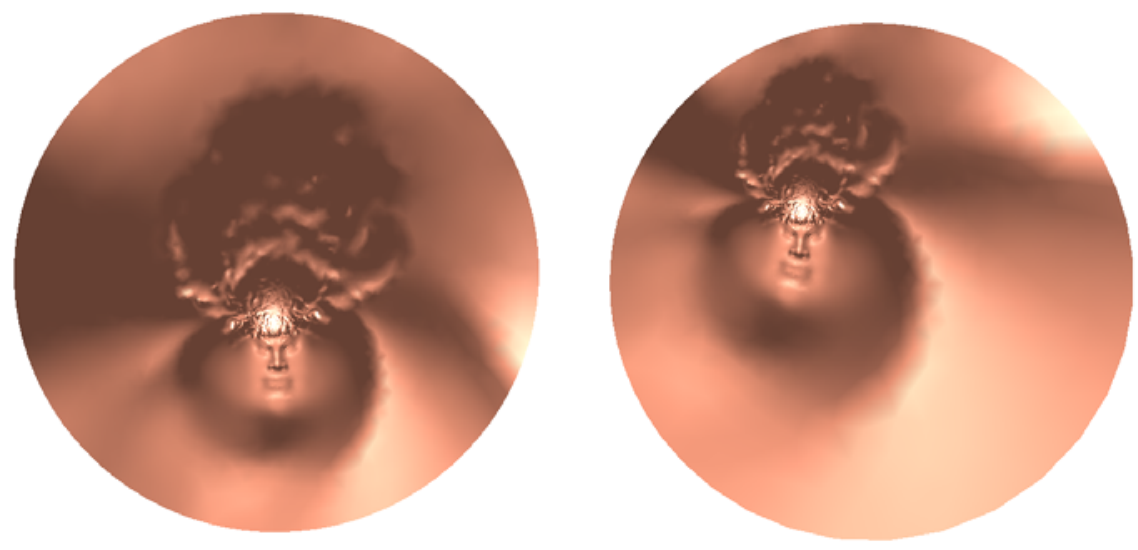

FIG. 3. Möbius transformation from the unit disk to itself.

tube shapes. Suppose we have a long thin cylinder and we conformally parameterize it. The center of the top is mapped to the origin. If we use polar coordinates $(\rho, \theta)$, then the conformal factor is a function dependent only on $\rho$ because of symmetry. The Gaussian curvature $K$ of the cylinder is zero, and

$$
K(\rho, \theta)=\frac{1}{\lambda^{2}} \Delta \log \lambda=0
$$

We can deduce $\lambda(\rho)=\exp (a \rho+b)$, where $a, b$ are constants. No matter what kind of conformal parameterizations we choose, the stretching is exponential. We have to change the topology of the surface by introducing a small boundary at the top of the cylinder and then the conformal factor becomes constant.

Base on this observation, we design our greedy topological modification algorithm as follows. First we find the most uniform conformal parameterization for current surface. Second, we locate points with extremely high conformal factors. Third, we introduce a small slice at the neighborhoods of those points. Finally, its conformal structure is recomputed. We repeat the whole process until the uniformity energy is less than some threshold.

Estimating Conformal Factor. Suppose we have obtained a global conformal parameterization induced by a holomorphic one-form $\omega$. The conformal factor for each vertex can be estimated by the following formula:

$$
\lambda(v)=\frac{1}{n} \sum_{\left[v_{i}, v\right] \in K_{1}} \frac{\left|r\left(v_{i}\right)-r(v)\right|^{2}}{\left|\omega\left(\left[v_{i}, v\right]\right)\right|^{2}}, v_{i}, v \in K_{0}
$$

where $n$ is the valence of vertex $v$. In practice, we compute $\frac{1}{\lambda}$ instead of computing itself. Then at the extreme points, the inverse of the conformal factor is very close to zero.

Locating the Extreme Points. We locate the cluster of vertices with relatively high conformal factors and compute their center of gravity, and find the closest vertex to it. This vertex 


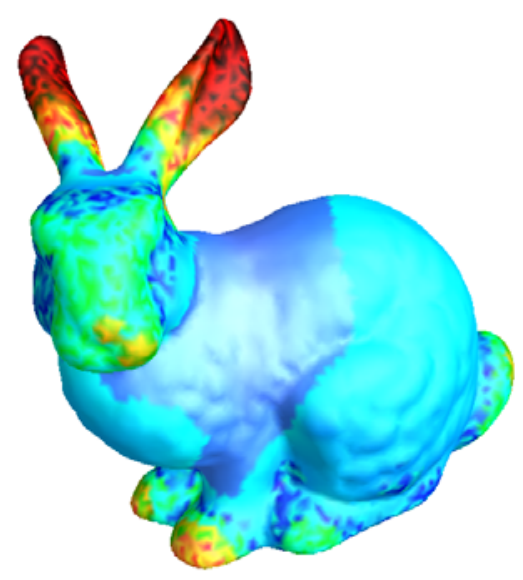

(a)

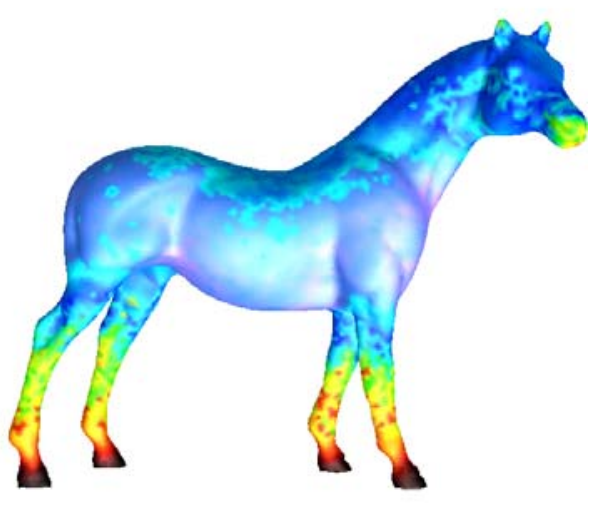

(b)

FIG. 4. Locating Extreme Point. Conformal factor is color encoded into bunny model (a). Conformal factor is color encoded into horse model $(b)$.

is an extreme point. Then we introduce a small slice through each extreme point, double covering the surface as described in [ Gu and Yau 2003], and compute a holomorphic 1-form basis. The optimal parameterization of current topology is computed by minimizing the uniformity energy. We repeat the whole procedure until the energy is smaller than a specified threshold or converges to a limit.

Then we need to address the question of whether the uniformity would really be improved by this procedure. Suppose at step $n$, we get a surface $S_{n}$. Then any global conformal parameterizations for $S_{n}$ is also a global conformal parameterization for $S_{n+1}$, and the minimal uniformity energy of $S_{n+1}$ denoted as $E_{n+1}$ is no greater than that of $S_{n}$. The sequence $\left\{E_{0}, E_{1}, E_{n+1}, \cdots\right\}$ is non-increasing and will converge to a limit. In practice, if the optimal uniformity energy does not decrease too much, the procedure will terminate.

The results for topological optimization are illustrated in Figure 4. In Figure 4(a), the bunny is conformally mapped to a sphere. The conformal factors are color encoded where the red color means high conformal factor. The tips of ears are located accurately. A horse model is also processed. The feet, the mouth and the tip of ears are regions with high conformal factor. Then we introduce small boundaries to them and compute conformal structure for the modified surfaces.

Suppose a closed genus zero surface has $k$ boundaries after topological optimization, its double covering is of genus $k-1$. The parameterization can be further optimized by the method for high genus surfaces. Although we introduce more zero points, the quality of the parameterization is improved greatly. The boundary of small slices will be mapped to an iso-parametric line in the parameter domain, no singularities are introduced along the slices. In theory, the slices could be as small as possible to avoid affecting the rendering effect. 
3.6. Zero Points Allocation. For a genus $g>1$ surface, there are $2 g-2$ zero points in a global conformal parameterization. In the neighborhood of zero points, the parameter areas of their neighborhoods are very small. If we want to construct geometry images from the surface, then these regions will be under-sampled. Then it is desirable to allocate zero points at some predetermined positions.

The positions of the zero points are globally related. They are determined by the conformal structure of the surface and are invariant under conformal mapping between surfaces. It is impossible to assign $2 g-2$ arbitrary points on the surface as the zero points.

Suppose $\omega$ is a holomorphic 1 -form, it has $p_{1}, p_{2}, \cdots, p_{2 g-2}$ as zero points, then $\omega\left(p_{i}\right)=$ $0, \forall i$. Let $\omega=\sum_{j=1}^{2 g} \lambda_{j} \omega_{j}$, then we get the linear system

$$
\sum_{j=1}^{2 g} \lambda_{j} \omega_{j}\left(p_{i}\right)=0, j=1,2, \cdots, 2 g-2
$$

If $\left\{p_{1}, p_{2}, \cdots, p_{2 g-2}\right\}$ is a set of zero points for some holomorphic one-form $\omega \neq 0$, it is necessary and sufficient that the matrix $\left(\omega_{j}\left(p_{i}\right)\right)$ is degenerated.

In our discrete setting, $\omega=\sum_{i=1}^{2 g} \lambda_{i} \omega_{i}$, and we use the following to approximate $\omega(v), v \in$ $K_{0}$.

$$
\omega(v)=\sum_{\left[v_{i}, v\right] \in K_{1}} \frac{\omega\left(\left[v_{i}, v\right]\right)}{\left|r\left(v_{i}\right)-r(v)\right|}=\sum_{\left[v_{i}, v\right] \in K_{1}} \sum_{j=1}^{2 g} \frac{\omega_{j}\left(\left[v_{i}, v\right]\right)}{\left|r\left(v_{i}\right)-r(v)\right|} .
$$

Suppose we want to set $n$ zero points $\left\{v_{1}, v_{2}, \cdots, v_{n}\right\}$, where $n<2 g-2$. Then we need to minimize the following energy

$$
E(\omega)=\sum_{i=1}^{n}\left|\omega\left(v_{i}\right)\right|^{2}
$$

This functional is a quadratic form of $\lambda_{1}, \lambda_{2}, \cdots, \lambda_{2 g}$ and can be solved easily using conjugate gradient method. If $n$ is not greater than $g$, then we can fix the zero points at the predetermined positions.

Figure 6 illustrates the two hole torus model. We predetermine the position of one zero point. By minimizing the energy in Equation 14, we can get the desired holomorphic one forms.

4. Results. The algorithms are developed using $\mathrm{C}++$ on Windows XP platform, and tested with a dual processor PC with main frequency 2.8G. The statistics are illustrated in Table 1 where all meshes are after topological optimization. We also tested the algorithm stability by optimizing parameters for bunny meshes with different resolutions. The simplified meshes are generated using progressive mesh method in [ Hoppe 1996]. The optimal parameterizations are consistent.

We test our algorithms on several surface models acquired by laser scanning. The bunny 


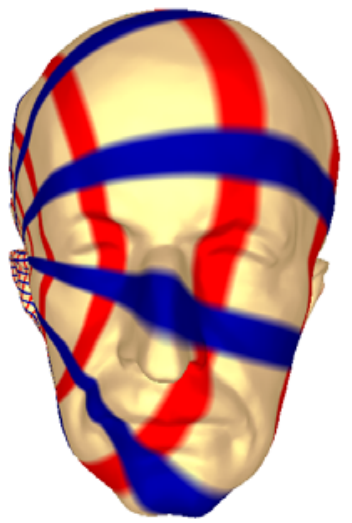

(a). Least Uniform, energy: 4.231e-5

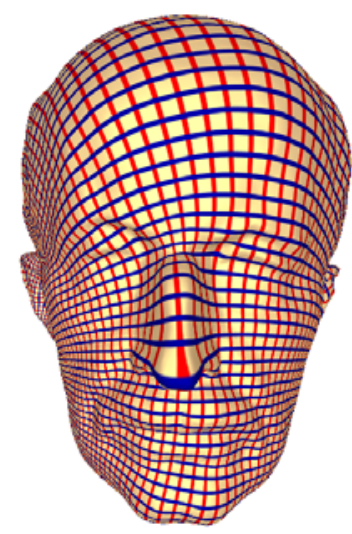

(b). Most Uniform, energy: $1.605 \mathrm{e}-5$

FIG. 5. Max Planck Head Model. Least and most uniform conformal parameterization.

model is of genus zero. The surface is sliced with 3 boundaries after topological optimization. The least uniform global conformal parameterization and most uniform global conformal parameterization results are illustrated in Figure 1 (a) and (b), respectively. Similarly, the horse model is of genus zero and it has 5 boundaries after topological modification. The least uniform global conformal parameterization and the most uniform global conformal parameterization are illustrated in Figure 1 (a) and (b), respectively.

The Max Planck head surface in Figure 5 is a topological disk. Figure 5(a) illustrates the result with minimum uniformity energy and Figure 5 (b) illustrates the result with maximum uniformity energy.

The human body surface in Figure 7 has 5 boundaries. The double covering of this surface is of genus 4 . We partition the whole surface to the left and right regions equally. The parameterization in Figure 7 (a) emphasizes the right region, which occupies $98.11 \%$ of the total parameter area. The parameterization in Figure 7 (b) emphasizes the left region, which occupies $96.1 \%$ of the total parameter area. The least uniform and the most uniform parameterization results are shown in Figure 7 (c) and (d) respectively.

Figure 6 illustrates the positions of zero points. We can get the desired holomorphic one forms by minimizing Equation 14. The Michelangelo's David surface is illustrated in Figure 8. We control the zero points' position using the method described in Section 3.6. In Figure 8(a), a zero point is located at the left upper arm near the shoulder. The same global conformal parameterization also has a zero point at his right upper arm near the shoulder. In Figure 8(b), there is a zero point under the left armpit. The same global conformal parameterization also gives a zero point at the right armpit, as shown in Figure 8(d).

5. Conclusion and Future Work. This work introduces systematic algorithms to optimize global conformal surface parameterizations. We define uniformity energy to measure 
TABLE 1

Performance for global conformal parameterization optimization.

\begin{tabular}{|c|c|c|c|c|}
\hline Mesh & Vertices & Genus & Boundaries & Time (s) \\
\hline eight & 766 & 2 & 0 & 30 \\
\hline bunny & 23996 & 0 & 3 & 150 \\
\hline horse & 19994 & 0 & 7 & 250 \\
\hline Max-Planck & 23609 & 0 & 1 & 180 \\
\hline Body & 40000 & 0 & 5 & 350 \\
\hline David & 200000 & 0 & 5 & 1800 \\
\hline
\end{tabular}

the uniformity of the parameterization. We define emphasized area energy to measure the parameter area of regions of interest. We also define special functional to allocate zero points at the desired positions. The problem of optimizing global conformal parameterizations is equivalent to searching for a desired Möbius transformation for genus zero surfaces and a desired holomorphic 1-form for high genus surfaces. We model global parameter optimizations as finite dimensional optimization problems, and use Newton's method to solve them. We also introduce algorithms to automatically modify the topology and allocate zero points at the specified positions to improve the quality of the global parameterization. The algorithms developed are efficient, intrinsic, practical, and versatile for different applications.

In the future, we will generalize the global conformal parameterizations to other parameterizations, such as Tuette, Stereo, Alexa parameterization as in [ Gotsman et al. 2003], and intrinsic parameterization as in [ Desbrun et al. 2002]. The generalization will be based on geometric differential equation theories. Then we will generalize the optimization methods in this work to these parameterization also.

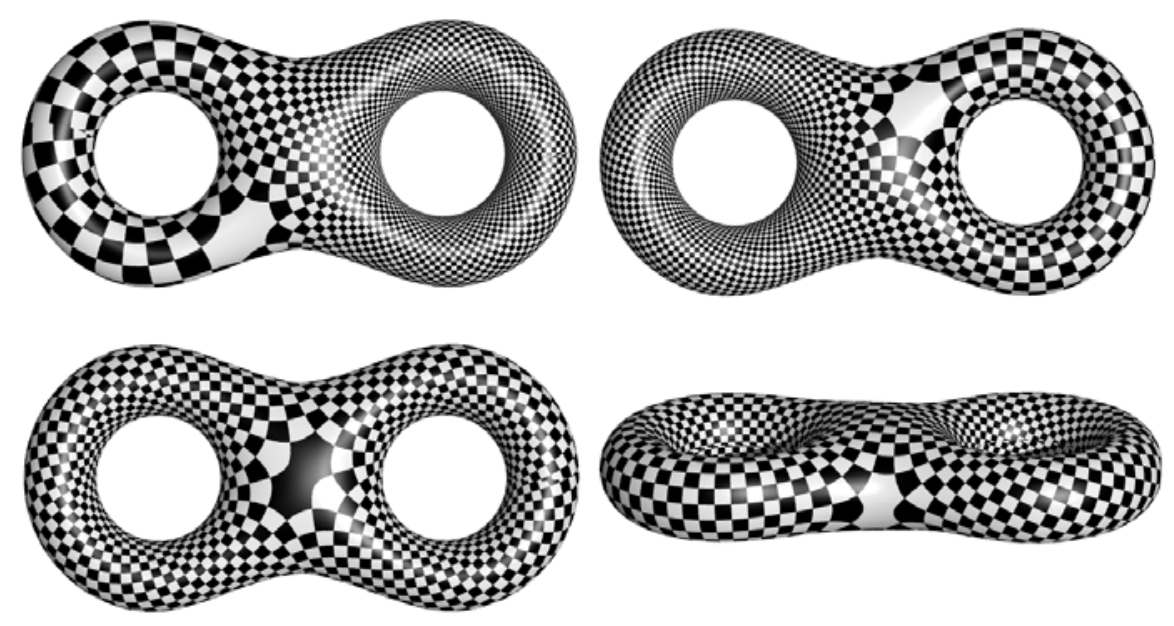

FIG. 6. Two hole torus Model. Locate zero points at different positions 


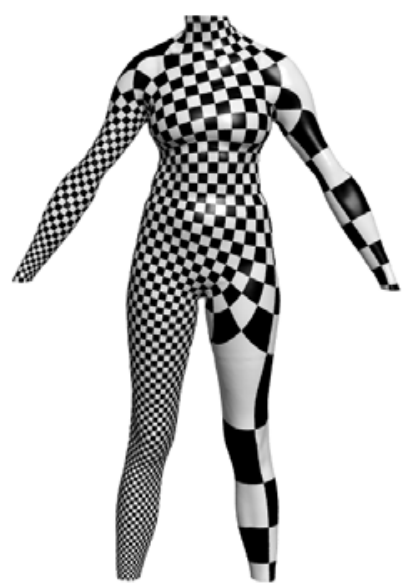

a. Emphasizing Left Part

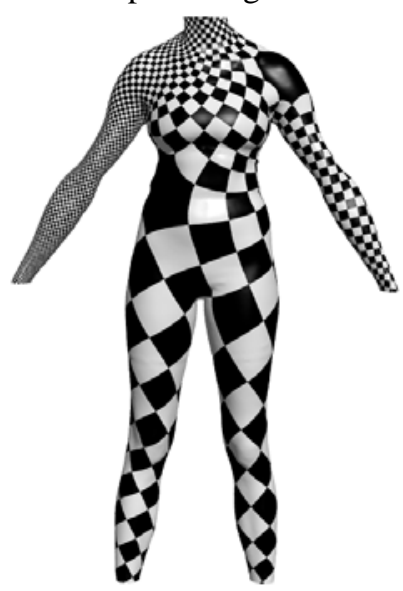

c. Least Uniform

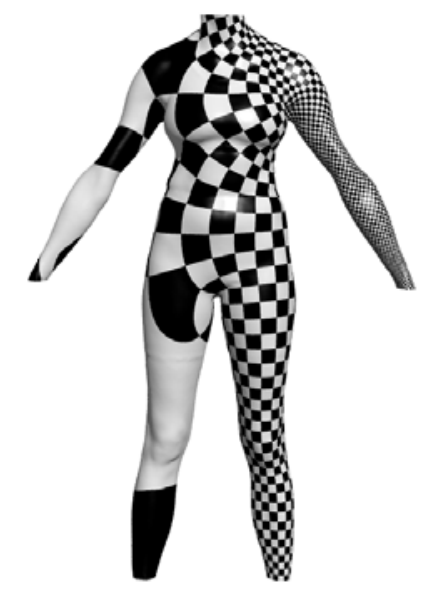

b. Emphasizing Right Part

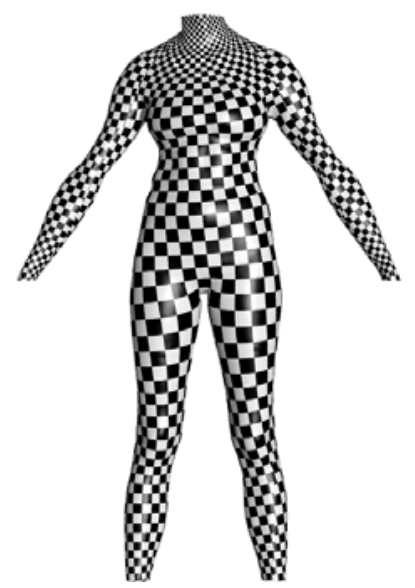

d. Most Uniform

FIG. 7. Human body Model. (a) Maximizing the parameter areas of left, percentage: $98.11 \%$. (b) Maximizing the parameter areas of right, percentage: $96.01 \%$. (c)Least uniform conformal parameterization, energy: 2.798 5(c). (d) Most uniform conformal parameterization, energy: 1.501e-5 (d).

\section{REFERENCES}

[ Alliez et al. 2002]

[ Angenent et al. 1999]

[ Ciarlet 1978]

[ Degener et al. 2003]

[ Desbrun et al. 2002]

[ Duchamp et al. 1997]
P. Alliez, M. Meyer, And M. Desbrun, Interactive geomety remeshing, In: SIGGRAPH 02, 2002, pp. 347-354.

S. Angenent, S. Haker, A. TAnnenaum, And R. Kikinis, Conformal geometry and brain flattening, MICCAI, 1999, pp. 271-278.

P. CIARLET, The finite element method for elliptic problems. North-Holland, Amsterdam, 1978.

P. Degener, J. Meseth, AND R. Klein, An adaptable surface parameterization method, In: Proceedings of the 12th International Meshing Roundtable, pp. 227237.

M. Desbrun, M. Meyer, AND P. Alliez. Intrinsic parametrizations of surface meshes, In: Proceedings of Eurographics, 2002.

T. Duchamp, A. Certian, A. Derose, And W. Stuetzle, Hierarchical compu- 


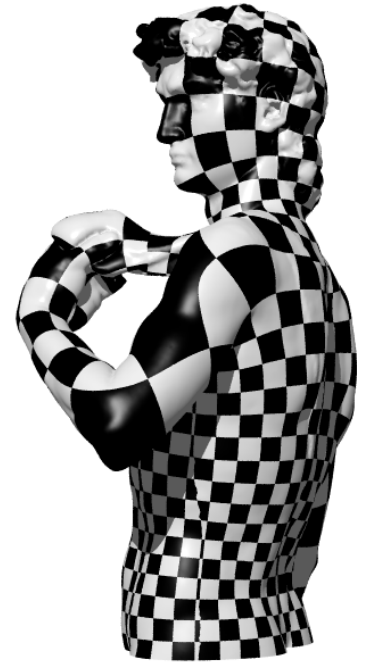

a. Zero Point at Left Shoulder

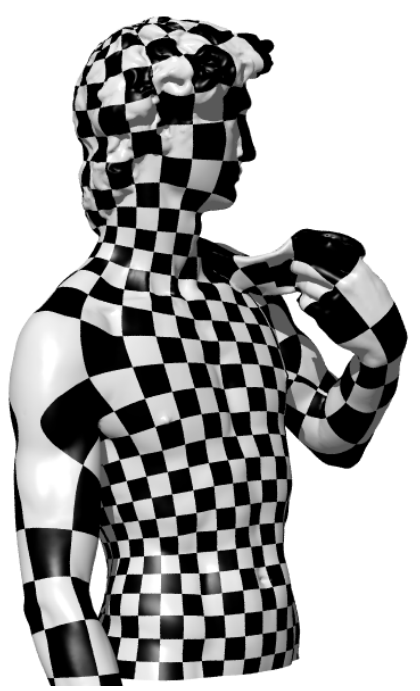

c. Zero Point at Right shoulder

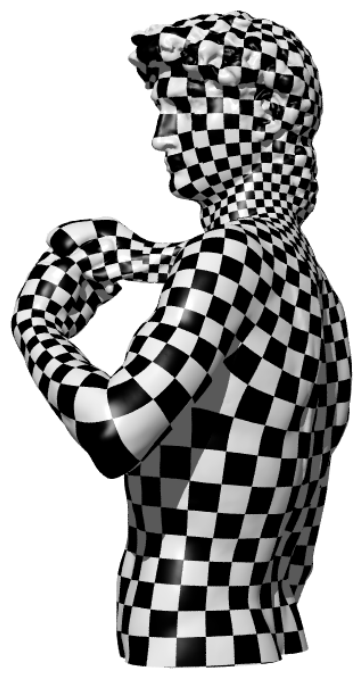

b. Zero Point at Left armpit

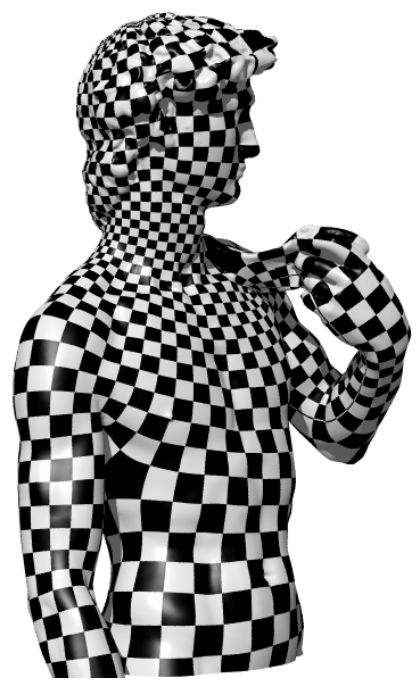

d. Zero Point at Right armpit

FIG. 8. Zero Point Allocation. Zero point is originally at left shoulder(a). Put zero point at Left armpit(b). Zero point is originally at right shoulder(c). Most uniform Put zero point at right armpit(d).

[ Eck et al. 1995]

tation of pl harmonic embeddings, preprint, 1997.

M. Eck, T. DeRose, T. Duchamp, H. Hoppe, M. Lounsbery, and W. StuetZLE, Multiresolution analysis of arbitrary meshes. In: SIGGRAPH 95 (1995), pp. 173-182.

[ Floater and Hormann 2003] M. FLOATER AND K. HormAnN, Recent advances in surface parameterization, In: Multiresolution in Geometric Modelling, 2003.

[ Floater 1997] M. FLOATER, Parameterization and smooth approximation of surface triangulations, In: Computer Aided Geometric Design, Vol. 14, 1997, pp. 231-250.

[ Gotsman et al. 2003]
C. Gotsman, X. Gu, AND A. ShefFer, Fundamentals of spherical parameterization for 3d meshes. In: ACM Transaction on Graphics, Vol. 22, 2003, pp. 
$358-363$.

[ Griffiths and Harris 1978] P. GRIfFITHS AND J. HARRIS, Principles of Algebraic Geometry, John Wiley and Sons, 1978.

[ Gu and Yau 2002] X. GU AND S. YAU, Computing conformal structures of surfaces, Communications in Information and Systems, 2:2(2002), pp. 121-146.

[ Gu and Yau 2003] X. GU AND S.-T. YAU, Global conformal surface parameterization, In: ACM Symposium on Geometry Processing, 2003, pp. 127-137.

[ Gu et al. 2003] X. Gu, Y. WANG, T. Chan, P. Thompson, And S.-T YAU, Genus zero surface conformal mapping and its application to brain surface mapping, In: Information Processing in Medical Imaging, 2003, 172-184.

[ Haker et al. 2000]

S. Haker, S. Angenent, A. Tannenbaum, R. Kikinis, G. SAPiro, and M. HALLE. Conformal surface parameterization for texture mapping, IEEE Transactions on Visualization and Computer Graphics, 6 (2000), 240-251.

[ Hoppe 1996]

H. Hoppe, Progressive meshes, In: Computer Graphics (Proceedings of SIGGRAPH 96), 1996, pp. 99-108.

[ Hormann and Greiner 1999] K. Hormann AND G. Greiner, Mips: An efficient global parameterization method, In: Curve and Surface Design: Saint-Malo 1999, Vanderbilt University Press, 1999, pp. 153-162.

[ Jost 1991]

J. Jost, Compact Riemann Surfaces: An Introduction to Contemporary Mathematics, Springer, 1991.

[ Kraevoy et al. 2003]

V. Kraevoy, A. Sheffer, And C. Gotsman, Matchmaker: Constructing constrained texture maps, In: SIGGRAPH 03 (2003), pp. 326-333.

[ Lévy et al. 2002]

B. LÉvy, S. PETITJEAN, N. RAY, AND J. MAILlot, Least squares conformal maps for automatic texture atlas generation, InSIGGRAPH 02 (2002), pp. 362-371.

[ Lévy 2003]

B. LÉVy, Dual domain extrapolation, In: SIGGRAPH 03 (2003), pp. 364-369.

[ Mercat 2001]

C. Mercat, Discrete Riemann surfaces and the Ising model, Comm. Math. Phys., 218:1(2001), pp. 177-216.

[ Pinkall and Polthier 1993] U. Pinkall AND K. Polthier, Computing discrete minimal surfaces and their conjugate, In: Experimental Mathematics, 2:1(1993), pp. 15-36.

[ Schoen and Yau 1997]

R. SCHOEN AND S. YAU, Lectures on Harmonic Maps. International Press, Harvard University, Cambridge MA, 1997.

[ Stephenson 1999] K. STEPHENSON, Approximation of conformal structures via circle packing, In: Computational Methods and Function Theory 1997, Proceedings of the Third CMFT conference, World Scientific, 1999, pp. 551-582. 